\title{
Three Dimensional Space-Time Gravitational Metric, 3 Space + 3 Time Dimensions
}

\author{
Espen Gaarder Haug \\ Norwegian University of Life Sciences, Oslo, Norway \\ Email: espenhaug@mac.com
}

How to cite this paper: Haug, E.G. (2021) Three Dimensional Space-Time Gravitational Metric, 3 Space +3 Time Dimensions. Journal of High Energy Physics, Gravitation and Cosmology, 7, 1230-1254. https://doi.org/10.4236/jhepgc.2021.74074

Received: July 13, 2021

Accepted: August 24, 2021

Published: August 27, 2021

Copyright $\odot 2021$ by author(s) and Scientific Research Publishing Inc. This work is licensed under the Creative Commons Attribution International License (CC BY 4.0).

http://creativecommons.org/licenses/by/4.0/

(c) (i) Open Access

\begin{abstract}
We have recently suggested a new quantum gravity theory that can be unified with quantum mechanics. We have coined this theory collision space-time. This new theory seems to be fully consistent with a 3-dimensional space-time, that is, three space dimensions and three time-dimensions, so some would call it six-dimensional. However, we have shown that collision-time and collision-length (space) are just two different sides of the same "coin" (spacetime), so it is more intuitive to think of them as 3-dimensional space-time. In previous papers, we have not laid out a geometric coordinate system for our theory that also considers gravity, but we will do that here. We are pointing out that Einstein's negative attitude towards relativistic mass can perhaps cause a weakness in the foundation of general relativity theory. When a relativistic mass is incorporated in the theory, this mass also seems to indicate one needs to move to three-dimensional space-time. Then, for example, our new theory matches fully up with all the properties of the Planck scale in relation to the mathematical properties of micro black holes, not only mathematically but also logically, something we demonstrate clearly that it is not the case of general relativity theory. Our new metric has many benefits as an alternative to the Schwarzschild metric and general relativity theory. It seems to be more consistent with the Planck units than the Schwarzschild metric. Most importantly, it seems to be fully consistent with a new quantum gravity theory that seems to unify gravity with quantum mechanics.
\end{abstract}

\section{Keywords}

Escape Velocity, Spherical Gravitational Metric, Schwarzschild Radius, Collision Space-Time, Planck Scale

\section{Background}

Today's standard physics is ruled by Minkowski [1] space-time which is the 
geometry of special relativity theory and flat space-time, and by several different metric systems such as the Schwarzschild metric when including gravity. These are four-dimensional space-time theories, where there are three space dimensions and one time dimension. However, our recent investigation indicates that such a four-dimensional space-time geometry not can be consistent with our new unified quantum gravity theory [2] [3]. We initially [4] tried to force our new theory into a four-dimensional space-time, but it seemed to lead to a few inconsistencies in some derivations that we first became fully aware of later. However, even in our first published paper on this topic, we suggest that a six-dimensional theory with three time dimensions and three space dimensions where possibly needed. To suggest a six-dimensional theory with three space and three time dimensions is not new, see [5]-[10]. Most of these papers were written in the $80 \mathrm{~s}$ and $90 \mathrm{~s}$ and are now more or less forgotten by most physicists, particularly since six dimensions did not seem to solve much and never got wider attention. However, with the invention of collision space-time, there are strong indications that three space and three time dimensions are needed. Therefore, we will suggest a space-time metric that is the "parallel" of the Schwarzschild metric under general relativity theory, while our new metric is compatible with collision space-time. However, we will claim that both general relativity theory and its Schwarzschild solution likely must be incomplete somehow. This point is one reason that has not been able to unify general relativity with quantum mechanics. Both quantum mechanics and standard gravity seem to be incomplete as they not can be unified likely. However, a few changes in Newtonian gravity combined with a few quantum mechanics changes that all come out from derivation from first principles about ideas on the origin of matter and energy in our new theory seem to lead to unification between quantum mechanics and quantum gravity.

An important point mentioned by Cole [11] is that, while $x, y$, and $z$ are observable separately, it is in four-dimensional space-time (Minkowski), that there only are one observable for the time measurement, $t$. One of the drawbacks of this viewpoint, compared to three time dimensions and three space dimensions, is as pointed out by Cole; that if the transformations for the six coordinates $x, y, z, t_{x}, t_{y}, t_{z}$ are linear, then the transformations for the quantities $x, y, z$ and $t$ become nonlinear. In other words, it seems to lead to a strong geometric simplification to add two time dimensions.

Four-dimensional space-time, as used in special relativity theory and several other relativity theories, has several shortcomings. Assume the standard Einstein light clock on board a train. If the train stands still, then we are in a rest-frame, and time can still move even if everything is at rest. Still, the light is moving. Light always moves in special relativity theory. Light is somehow something outside the rest-frame system, so to say. But if we look at the light, then light needs space to move in even if we are in a rest-frame. That is, to have time, we need space. Not only that, we need to move in space. But the link between photons 
and mass is not fully understood in standard theory, even if they had great progress there with Einstein's special relativity theory that gave us a hint in his famous equation $E=m c^{2}$, that light and mass was connected somehow. Still, standard physics is not even sure if a photon can have rest-mass or not. See [12] for a review article on this topic. In our theory, a collision between two light particles is the only pure mass, and the collision stands still. It is at absolute rest, see [2] [3], it takes up spatial dimensions as the light particles are similar to that suggested by Newton, namely indivisible and with spatial dimension, actually they have a diameter equal to the Planck length. That they have a diameter equal to the Planck length is not an assumption, this is what we find after calibrating our model to gravity observations. We will kindly ask the reader to study the papers just mentioned to grasp this issue. Here our focus is on the space-time geometry related to the gravity in our theory.

Several six-dimensional space plus time theories that have been suggested in the past suggest superluminal signal interpretations. However, there are no superluminal signals in our theory, except for such things as mutual velocity where also special relativity has speeds above $c$, see, for example, Sommerfeld and Rindler [13] [14]. Also, some suggested six-dimensional space-time theories have, for example, five space dimensions and one time dimension. Our theory is a three time dimension, three space dimension theory.

\section{General Relativity Theory and the Schwarzschild Metric}

We will shortly mention the Schwarzschild [15] [16] solution to Einstein's general relativity theory [17], so one more easily can compare our new suggested collision space-time metric with this established "consensus" theory. There is a very strong link between the Schwarzschild solution and escape velocity. The escape velocity for general relativity is given by

$$
v_{e}=\sqrt{\frac{G M}{r^{2}}} .
$$

We can actually express the Schwarzschild solution in the form of the escape velocity. This equation gives

$$
\begin{aligned}
& g=-c^{2} \mathrm{~d} \tau^{2}=-\sqrt{1-\frac{v_{e}^{2}}{c^{2}}} c^{2} \mathrm{~d} t^{2}+\frac{\mathrm{d} r^{2}}{{\sqrt{1-\frac{v_{e}^{2}}{c^{2}}}}^{2}}+r^{2} \mathrm{~d} \theta^{2}+r^{2} \sin \theta \mathrm{d} \phi^{2}
\end{aligned}
$$

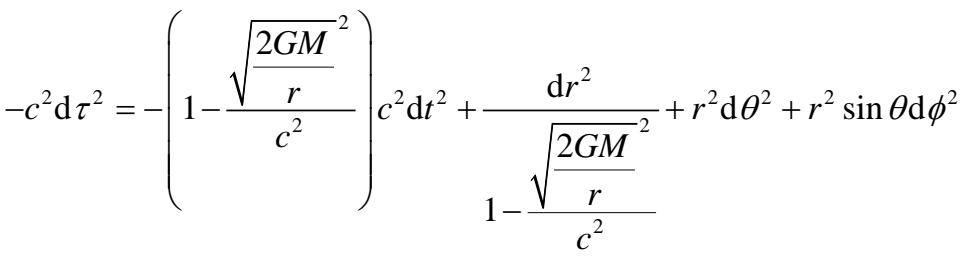

$$
\begin{aligned}
& -c^{2} \mathrm{~d} \tau^{2}=-\left(1-\frac{2 G M}{r c^{2}}\right) c^{2} \mathrm{~d} t^{2}+\frac{\mathrm{d} r^{2}}{1-\frac{2 G M}{r c^{2}}}+r^{2} \mathrm{~d} \theta^{2}+r^{2} \sin \theta \mathrm{d} \phi^{2}
\end{aligned}
$$




$$
-c^{2} \mathrm{~d} \tau^{2}=-\left(1-\frac{r_{s}}{r}\right) c^{2} \mathrm{~d} t^{2}+\frac{\mathrm{d} r^{2}}{1-\frac{r_{s}}{r}}+r^{2} \mathrm{~d} \theta^{2}+r^{2} \sin \theta \mathrm{d} \phi^{2}
$$

This result is the well-known Schwarzschild solution. We have not shown a derivation of it here, but simply shown how it can be expressed in combination with the escape velocity. A key point here is that the escape velocity that one gets from general relativity theory is identical to that one gets from standard nonrelativistic Newton mechanics, see [18] [19]. The Newtonian escape velocity is derived by solving the following equation with respect to $V$

$$
\frac{1}{2} m v^{2}-\frac{G M m}{r}=0
$$

which gives $v_{e}=\sqrt{\frac{G M}{r^{2}}}$. Pay attention to that one here has used a non-relativistic kinetic energy approximation in the derivation because $\frac{1}{2} m v^{2}$ is the first Taylor series expansion term one get from the relativistic kinetic energy of Einstein $E_{k}=m c^{2} \gamma-m c^{2}$. The first term of the Taylor expansion is only a good approximation when $v \ll c$. For example, when the escape velocity is close to $c$, this derivation and escape velocity clearly cannot be used. Second, the small mass that is moving with velocity $v$ has no adjustments for relativistic mass in the Newton gravitational energy potential formula $G \frac{M m}{r}$. The Newton derivation that leads to the escape velocity derived from Newtonian mechanics does not consider relativistic kinetic energy or relativistic mass. One can wonder then how general relativity theory that should consider all relativistic effects can give the same formula, but this is the case, clearly demonstrated by [18]. We suspect something has gone wrong during the way or the foundation of the theory. We suspect one possible error has been to incorporate standard Newton theory as a weak field limit. For a massive gravitational body, the gravitational acceleration field at the Schwarzschild radius is very weak. However, at the same time, the escape velocity is $c$ there, that is $m$ when close to this radius moves at speeds close to $c$, the Newton escape velocity is then clearly not valid. Further, one has mistakenly assumed Newton's theory is consistent with infinite gravity speed, a point that has recently been questioned [20]. Standard Newton theory seems to be consistent with that gravity moves at the speed of light (standard physics here assume infinite speed), but at the same time, it assumes the small mass $m$ is moving very slow relative to $c$. We simply think standard physics has not understood Newton's gravitational theory fully in relation to its strength and limitation. By incorporating Newton as a weak field limit, their theory is not fully consistent. We will, in the section below, come back to this and a possible solution.

\section{6-Dimensional Space-Time Geometry}

We [2] [4] have recently suggested a quantum gravity theory that unifies gravity and quantum mechanics and simplify many things in physics. The latest version 
of our theory strongly indicates we must have a three-dimensional space-time to get a fully consistent theory. Time and space are, in our theory, two different sides of the same coin. One cannot move in space without moving in time (as also thought in standard theory), but we cannot move in time without moving in space because time in our theory is always linked to photon clocks that are again linked to mass. Mass at the deepest level in our theory is simply collisions between two photons, and a collision is also a tick of time. For the clock to tick, the photons need to move and collide again. Inside an elementary particle, the photons move back and forth over the particle's reduced Compton wavelength and collide. The possibility that mass is linked to Compton clocks has some support from other independent research also, see [21] [22].

At the deepest level of the quantum world, we cannot move, for example, only in the $x$ direction off space and at the same time in the $t_{y}$ direction of time. If we move only in the $x$ direction of space, we must also move in the $t_{x}$ direction in time. Also, we cannot move, for example, in the $t_{y}$ direction of time without also moving in the $y$ direction of space again because the building blocks of matter are photons. That is, space and time are basically two sides of the same coin. This point we have described in some detail in [2], here we will extend this three-dimensional space-time geometry $(3+3)$ to also hold for gravity. We suggest we must have the following space-time metric (Cartesian coordinate system and flat space-time)

$$
\mathrm{d} s^{2}=-c^{2} \mathrm{~d} t_{x}^{2}-c^{2} \mathrm{~d} t_{y}^{2}-c^{2} \mathrm{~d} t_{z}^{2}+\mathrm{d} x^{2}+\mathrm{d} y^{2}+\mathrm{d} z^{2}
$$

in the special case for the Planck mass particle, this case can be simplified to this equation as the indivisible particle stands still when colliding or move at the speed of light when not colliding, that is

$$
0=-c^{2} \mathrm{~d} t_{x}^{2}-c^{2} \mathrm{~d} t_{y}^{2}-c^{2} \mathrm{~d} t_{z}^{2}+\mathrm{d} x^{2}+\mathrm{d} y^{2}+\mathrm{d} z^{2} .
$$

This result should come as no surprise as already in 1960, Rindler [23] showed that the Minkowski space-time could be simplified from

$-\mathrm{d} t^{2} c^{2}+\mathrm{d} x^{2}+\mathrm{d} y^{2}+\mathrm{dz} z^{2}=\mathrm{d} s^{2}$ to $-\mathrm{d} t^{2} c^{2}+\mathrm{d} x^{2}+\mathrm{d} y^{2}+\mathrm{d} z^{2}=0$ when dealing strictly with light signals, see also [2]. Further also in the moving system $-\mathrm{d} t^{\prime 2} c^{2}+\mathrm{d} x^{\prime 2}+\mathrm{d} y^{\prime 2}+\mathrm{d} z^{\prime 2}=0$, see also [24]. We can also express our 6-D space-time metric on matrix form

$$
\eta_{\mu, v}=\left(\begin{array}{llllll}
\eta_{0,0} & \eta_{0,1} & \eta_{0,2} & \eta_{0,3} & \eta_{0,4} & \eta_{0,5} \\
\eta_{1,0} & \eta_{1,1} & \eta_{1,2} & \eta_{1,3} & \eta_{1,4} & \eta_{1,5} \\
\eta_{2,0} & \eta_{2,1} & \eta_{2,2} & \eta_{2,3} & \eta_{2,4} & \eta_{2,5} \\
\eta_{3,0} & \eta_{3,1} & \eta_{3,2} & \eta_{3,3} & \eta_{3,4} & \eta_{3,5} \\
\eta_{4,0} & \eta_{4,1} & \eta_{4,2} & \eta_{4,3} & \eta_{4,4} & \eta_{4,5} \\
\eta_{5,0} & \eta_{5,1} & \eta_{5,2} & \eta_{5,3} & \eta_{5,4} & \eta_{5,5}
\end{array}\right)=\left(\begin{array}{cccccc}
-1 & 0 & 0 & 0 & 0 & 0 \\
0 & -1 & 0 & 0 & 0 & 0 \\
0 & 0 & -1 & 0 & 0 & 0 \\
0 & 0 & 0 & 1 & 0 & 0 \\
0 & 0 & 0 & 0 & 1 & 0 \\
0 & 0 & 0 & 0 & 0 & 1
\end{array}\right) .
$$

Which again can be represented as

$$
\mathrm{d} s^{2}=\sum_{\mu=0}^{5} \sum_{v=0}^{5} \eta_{\mu, v} \mathrm{~d} x^{\mu} \mathrm{d} x^{v}
$$




$$
\mathrm{d} s^{2}=-c^{2} \mathrm{~d} t_{x}^{2}-c^{2} \mathrm{~d} t_{y}^{2}-c^{2} \mathrm{~d} t_{z}^{2}+\mathrm{d} x^{2}+\mathrm{d} y^{2}+\mathrm{d} z^{2} .
$$

When it comes to adding gravity, we know that our escape velocity is [4]

$$
v_{e}=\sqrt{\frac{2 G M}{r}-\frac{G^{2} M^{2}}{r^{2} c^{2}}} .
$$

This result can simply be derived from relativistic modified Newton. That is, we solve the following equation with respect to $V$

$$
\begin{gathered}
\frac{m c^{2}}{\sqrt{1-\frac{v^{2}}{c^{2}}}}-m c^{2}-\frac{G M \frac{m}{\sqrt{1-\frac{v^{2}}{c^{2}}}}}{r}=0 \\
1-\sqrt{1-\frac{v^{2}}{c^{2}}}-\frac{G M}{c^{2} r}=0 \\
\sqrt{1-\frac{v^{2}}{c^{2}}}=-\frac{G M}{c^{2} r}+1 \\
1-\frac{v^{2}}{c^{2}}=\left(-\frac{G M}{c^{2} r}+1\right)^{2} \\
1-\frac{v^{2}}{c^{2}}=\left(-\frac{G^{2} M^{2}}{c^{4} r^{2}}+\frac{2 G M}{c^{2} r}\right)^{2}+1 \\
v=\sqrt{\frac{2 G M}{r}-\frac{G^{2} M^{2}}{c^{2} r^{2}}} .
\end{gathered}
$$

This in contrast to general relativity that gives an escape velocity of $v_{e}=\sqrt{\frac{2 G M}{r}}$. Here we are also at one of the possible mistakes done in the foundation of general relativity theory. Already in 1904, Lorentz had the formula for relativistic mass correct, as we know it today, namely $m_{r}=\gamma m$, where $m$ is the rest mass, and $\gamma=1 / \sqrt{1-v^{2} / c^{2}}$, and $m_{r}$ is the relativistic mass. Einstein in 1905 was not aware of Lorentz 1904 paper and published his own formulas for relativistic mass at the end of his famous papers, his formulas where

$$
\text { longitudinal mass }=m \gamma^{3}=\frac{m}{\sqrt{1-\frac{v^{2}}{c^{2}}}}
$$

and

$$
\text { transverse mass }=m \gamma^{2}=\frac{m}{1-\frac{v^{2}}{c^{2}}} .
$$

Also, Lorentz had suggested longitudinal and transverse mass and the same longitudinal mass as Einstein, but Lorentz transverse mass is what many researchers today call relativistic mass [25] [26] [27]. Max Born in 1920 [28] was likely the first to coin $m_{r}=\gamma m$ for "relativistic mass". Einstein could have the 
correct relativistic mass formula simply by dividing his relativistic energy formula $E=m c^{2} \gamma$ by $c^{2}$. However, he had not done that and instead derived what it is full agreement on today, at least not a correct relativistic mass formula. The relativistic mass leads to strange interpretations in four-dimensional space-time, and Einstein after a few more years abandoned relativistic mass totally. We should also keep in mind that other relativity theories were considered still competitors of Einstein's special relativity theory, particularly the Lorentz interpretation of relativity, so we can also speculate if Einstein, for this reason, was extra negative to relativistic mass. A series of researchers have strongly criticized the use of relativistic mass and that it should not be used. See for example [29] [30] [31] [32]. For example, Adler has claimed:

Anyone who has tried to teach special relativity using the four-vector space-time approach knows relativistic mass and four-vectors make for an ill-conceived marriage. In fact, most of the recent criticism of relativistic mass is presented in the context of the four-vector formulation of special relativity. - Adler 1987

In a letter to Lincoln Barnett, an American journalist, dated 19 June 1948, Einstein wrote,

It is not good to introduce the concept of the mass $M=m / \sqrt{1-\frac{v^{2}}{c^{2}}}$ of a moving body for which no clear definition can be given. It is better to introduce no other mass concept than the "rest mass" m. Instead of introducing $M$, it is better to mention the expression for the momentum and energy of a body in motion.

This claim by Einstein has fueled critics of the relativistic mass concept. See, for example, Hecht [31]. Still others also well studied in special relativity have defended the relativistic mass concept, see for example Rindler [14] [33] and Jammer [34]. We agree that the relativistic mass interpretation does not seem to fit well within Minkowski space-time and the four-vector approach. However, in three-dimensional space-time, it seems to fit in very well. This is why we have to incorporate relativistic mass in the Newton formula as we have done. Again, this fit leads to a new escape velocity formula, as mentioned above.

Combining our new escape velocity formula with the assumption that we must have three space and three time dimensions to be consistent with collision space-time, we will likely end up with the following metric (based on spherical polar coordinates)

$$
\begin{aligned}
\mathrm{d} s^{2}= & -\left(1-\frac{2 G M}{r c^{2}}+\frac{G^{2} M^{2}}{c^{4} r^{2}}\right) c^{2} \mathrm{~d} t^{2}-c^{2} t^{2} \mathrm{~d} \theta^{2}-c^{2} t^{2} \sin ^{2} \theta \mathrm{d} \phi^{2} \\
& +\frac{\mathrm{d} r^{2}}{1-\frac{2 G M}{r c^{2}}+\frac{G^{2} M^{2}}{c^{4} r^{2}}}+r^{2} \mathrm{~d} \theta^{2}+r^{2} \sin ^{2} \theta \mathrm{d} \phi^{2}
\end{aligned} .
$$


In the case that $t=\frac{r}{c}$, the equation above simplifies to

$$
\mathrm{d} s^{2}=-\left(1-\frac{2 G M}{r c^{2}}+\frac{G^{2} M^{2}}{c^{4} r^{2}}\right) c^{2} \mathrm{~d} t^{2}+\frac{\mathrm{d} r^{2}}{1-\frac{2 G M}{r c^{2}}+\frac{G^{2} M^{2}}{c^{4} r^{2}}} .
$$

That we can simplify using this procedure seems very reasonable. At a given radius $r$ in a spherical coordinate system, all points should be equal in terms of gravitational effects. Does one even, in general relativity theory have a single predictable phenomena according to the Schwarzschild metric that is also observable where one also needs the angle $\theta$ or $\phi$ ? Not to our knowledge, but we would be happy to hear comments or discussions around this point, we could be wrong, in that case the last simplification need to be re-considered.

Bear in mind that the gravitational constant likely is a composite constant of the form $G=\frac{l_{p}^{2} c^{3}}{\hbar}$ as suggested by [35] [36]. This result would lead to a circular problem if we not can find the Planck length independent of $G$, but recent findings have shown that we easily can find the Planck length with no knowledge of $G$, see for example [20] [37] [38] so this concern is not an issue. Further, any mass in terms of a kilogram can be expressed as

$$
m=\frac{\hbar}{\bar{\lambda}} \frac{1}{c}
$$

where $\bar{\lambda}$ is the reduced Compton wavelength [39]. This result is simply the Compton wavelength formula $\bar{\lambda}=\frac{\hbar}{m c}$ solved with respect to $m$. Our escape velocity formula can therefore also be expressed as

$$
v_{e}=\sqrt{\frac{2 G M}{r}-\frac{G^{2} M^{2}}{r^{2} c^{2}}}=\sqrt{\frac{2 c^{2} l_{p}^{2}}{\bar{\lambda} r}-\frac{c^{2} l_{p}^{4}}{\bar{\lambda}^{2} r^{2}}} .
$$

This equation also corresponds to the version of this escape velocity formula as given by [4]. This point means our metric also can be written in the form

$$
\begin{aligned}
\mathrm{d} s^{2}= & -\left(1-\frac{2 l_{p}^{2}}{\bar{\lambda} r}+\frac{l_{p}^{4}}{\bar{\lambda}^{2} r^{2}}\right) c^{2} \mathrm{~d} t^{2}-c^{2} t^{2} \mathrm{~d} \theta^{2}-c^{2} t^{2} \sin ^{2} \theta \mathrm{d} \phi^{2} \\
& +\frac{\mathrm{d} r^{2}}{1-\frac{2 l_{p}^{2}}{\bar{\lambda} r}+\frac{l_{p}^{4}}{\bar{\lambda}^{2} r^{2}}}+r^{2} \mathrm{~d} \theta^{2}+r^{2} \sin ^{2} \theta \mathrm{d} \phi^{2}
\end{aligned}
$$

In the case that $t=\frac{r}{c}$ simplifies to

$$
\mathrm{d} s^{2}=-\left(1-\frac{2 l_{p}^{2}}{\bar{\lambda} r}+\frac{l_{p}^{4}}{\bar{\lambda}^{2} r^{2}}\right) c^{2} \mathrm{~d} t^{2}+\frac{\mathrm{d} r^{2}}{1-\frac{2 l_{p}^{2}}{\bar{\lambda} r}+\frac{l_{p}^{4}}{\bar{\lambda}^{2} r^{2}}} .
$$

Therefore, it is clear from the metric itself that it is linked to the Planck scale. By re-writing $G$ and $M$ in the same way as mentioned above also in the 
Schwarzschild solution, one can also see that it is linked to the Planck scale, but we have reason to think general relativity and its Schwarzschild solution still is incomplete.

We can also write our new metric as

$$
\begin{aligned}
\mathrm{d} s^{2}= & -\left(1-\frac{2 c \bar{M}}{r}+\frac{c^{2} \bar{M}^{2}}{r^{2}}\right) c^{2} \mathrm{~d} t^{2}-c^{2} t^{2} \mathrm{~d} \theta^{2}-c^{2} t^{2} \sin ^{2} \theta \mathrm{d} \phi^{2} \\
& +\frac{\mathrm{d} r^{2}}{1-\frac{2 c \bar{M}}{r}+\frac{c^{2} \bar{M}^{2}}{r^{2}}}+r^{2} \mathrm{~d} \theta^{2}+r^{2} \sin ^{2} \theta \mathrm{d} \phi^{2}
\end{aligned}
$$

where $\bar{M}=\frac{l_{p}}{\bar{\lambda}} \frac{l_{p}}{c}$ is the collision-time mass, which is much more complete than the $\mathrm{kg}$, mass, but the $\mathrm{kg}$ mass is also fixed through multiplication with $G$, but this fact has not been understood by standard physics. In the case that $t=\frac{r}{c}$ then the equation above simplifies to

$$
\mathrm{d} s^{2}=-\left(1-\frac{2 c \bar{M}}{r}+\frac{c^{2} \bar{M}^{2}}{r^{2}}\right) c^{2} \mathrm{~d} t^{2}+\frac{\mathrm{d} r^{2}}{1-\frac{2 c \bar{M}}{r}+\frac{c^{2} \bar{M}^{2}}{r^{2}}} .
$$

\section{Tensor Form}

With coordinates $\left(x^{1}, x^{2}, x^{3}, x^{4}, x^{5}, x^{6}\right)=(c t, \theta, \phi, r, \theta, \phi)$ we can also write our metric theory on tensor form

$$
g_{\mu, v}=\left(\begin{array}{cccccc}
-\left(1-\frac{2 G M}{r c^{2}}+\frac{G^{2} M^{2}}{c^{4} r^{2}}\right) & 0 & 0 & 0 & 0 & 0 \\
0 & -c^{2} t^{2} & 0 & 0 & 0 \\
0 & 0 & -c^{2} t^{2} \sin ^{2} \theta & 0 & 0 \\
0 & 0 & 0 & \left(1-\frac{2 G M}{r c^{2}}+\frac{G^{2} M^{2}}{c^{4} r^{2}}\right)^{-1} & 0 & 0 \\
0 & 0 & 0 & 0 & r^{2} & 0 \\
0 & 0 & 0 & 0 & 0 & r^{2} \sin ^{2} \theta
\end{array}\right)
$$

or alternatively as

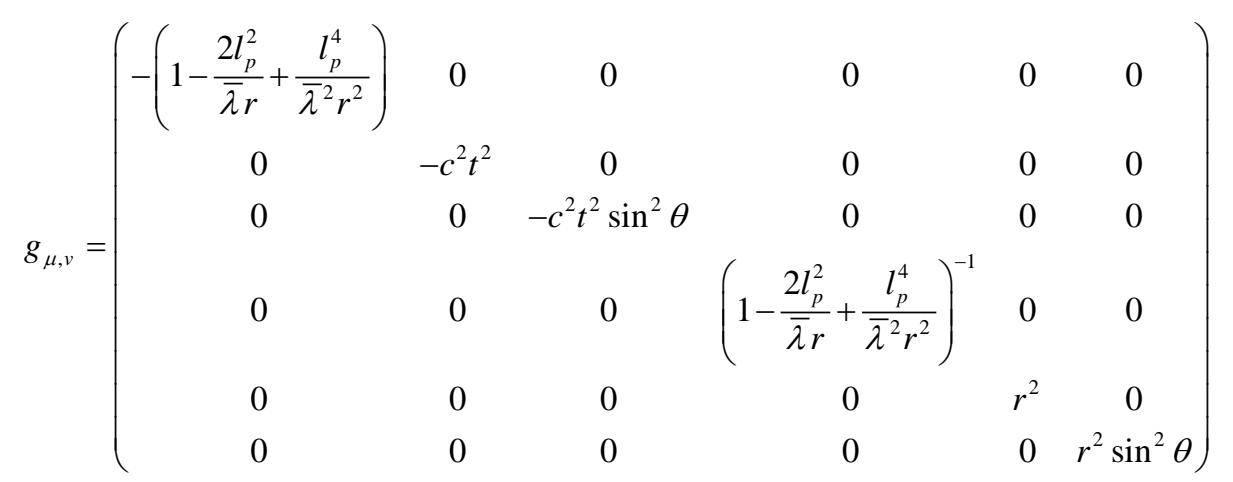


or alternatively as

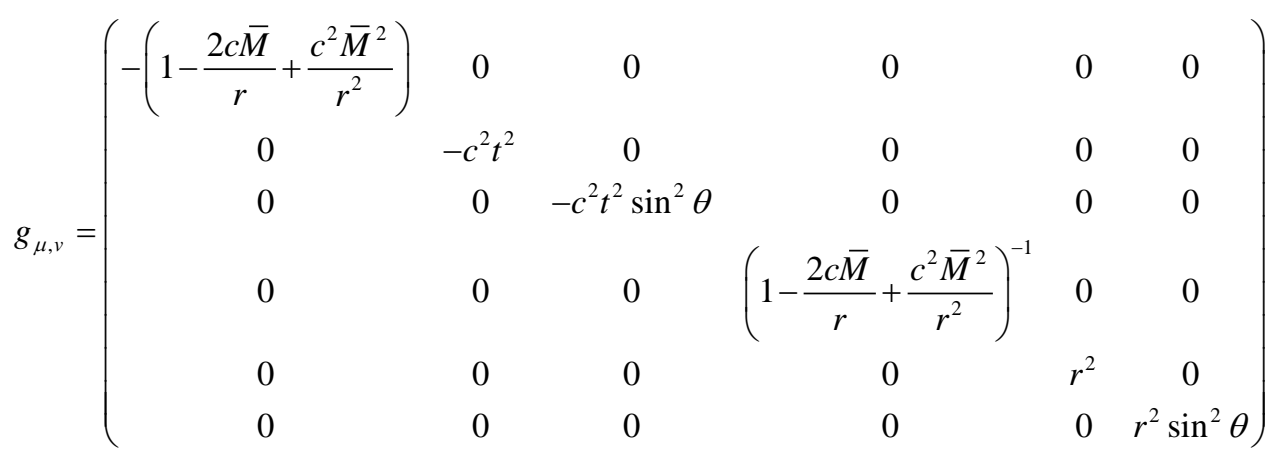

In the special case where $v_{e}=c$, then we have $r=\frac{G M}{c^{2}}$, that is we have an escape velocity of $c$ at half the Schwarzschild radius $r_{s}=\frac{2 G M}{c^{2}}$, then we have $g_{0,0}=0$ and $g_{3,3}=\infty$ will converges to infinity. That is also our metric has a singularity at $r$ when the escape velocity is $c$. However, we do not have a second singularity as there is no meaning in our theory to have $r=0$ as the Planck length is the smallest possible length, so we must have $r \geq l_{p}$ in our theory, see [4] for a discussion on this.

In the case $t=\frac{r}{c}$ these tensors can be simplified to

$$
g_{\mu, v}=\left(\begin{array}{cc}
-\left(1-\frac{2 G M}{r c^{2}}+\frac{G^{2} M^{2}}{c^{4} r^{2}}\right) & 0 \\
0 & \left(1-\frac{2 G M}{r c^{2}}+\frac{G^{2} M^{2}}{c^{4} r^{2}}\right)^{-1}
\end{array}\right) .
$$

This tensor corresponds to the metric Equation (12). Alternatively, we can write the tensor as

$$
g_{\mu, v}=\left(\begin{array}{cc}
-\left(1-\frac{2 l_{p}^{2}}{\bar{\lambda} r}+\frac{c l_{p}^{4}}{\bar{\lambda}^{2} r^{2}}\right) & 0 \\
0 & \left(1-\frac{2 l_{p}^{2}}{\bar{\lambda} r}+\frac{l_{p}^{4}}{\bar{\lambda}^{2} r^{2}}\right)^{-1}
\end{array}\right) .
$$

This tensor corresponds to the metric Equation (16). Alternatively, we can write the tensor as

$$
g_{\mu, v}=\left(\begin{array}{cc}
-\left(1-\frac{2 c \bar{M}}{r}+\frac{c^{2} \bar{M}^{2}}{r^{2}}\right) & 0 \\
0 & \left(1-\frac{2 c \bar{M}}{r}+\frac{c^{2} \bar{M}^{2}}{r^{2}}\right)^{-1}
\end{array}\right) .
$$

This tensor corresponds to the metric Equation (18). 


\section{Some Predictions from Our Metric and Our New Escape Velocity}

We will here present some predictions from our theory. Our escape velocity is given by

$$
v_{e}=\sqrt{\frac{2 G M}{r}-\frac{G^{2} M^{2}}{c^{2} r}}
$$

by setting $v_{e}=c$ and solving with respect to $r$ we get

$$
r_{c}=\frac{G M}{c^{2}} .
$$

This result is half of that of the Schwarzschild radius $r_{s}=\frac{2 G M}{c^{2}}$. In the special case where $v_{e}=c$ then the orbital velocity is identical to the escape velocity, that is

$$
v_{e}=\sqrt{\frac{2 G M}{r_{c}}-\frac{G^{2} M^{2}}{c^{2} r_{c}}}=\sqrt{\frac{2 G M}{r_{c}}}=v_{\text {orbit }} .
$$

This point means that in our theory, the radius of the photon sphere is identical to that of the radius where the escape velocity is $c$, unlike the Schwarzschild metric where the radius of the photon sphere is $\frac{3}{2} r_{s}$.

Gravitational time-dilation in our metric must be given by

$$
t_{0}=t_{f} \sqrt{1-\frac{2 G M}{c^{2} r}+\frac{G^{2} M^{2}}{c^{4} r^{2}}}
$$

as long as we are far away from the radius where the escape velocity is $c$ then this result gives a likely indistinguishable prediction from general relativity, close to the Schwarzschild radius or close to our new $r_{c}$ radius, the difference in prediction is large, so the main difference is in how to interpret so-called black holes, that in our theory have a quite different interpretation, something we come back to in the next section.

Gravitational red-shift is in our theory given by

$$
z=\frac{\lambda_{2}-\lambda_{1}}{\lambda_{1}}=\frac{\sqrt{1-\frac{2 G M}{c^{2} r_{1}}+\frac{G^{2} M^{2}}{c^{4} r_{1}^{2}}}}{\sqrt{1-\frac{2 G M}{c^{2} r_{2}}+\frac{G^{2} M^{2}}{c^{4} r_{2}^{2}}}}-1 .
$$

Also, here the predictions are indistinguishable from general relativity theory when far away from the Schwarzschild radius, but not when close.

\section{Micro Black Holes Fit the Planck Scale Very Well in Our New Theory, Not So Well in General Relativity Theory}

The Planck scale consists of the Planck mass, the Planck length and the Planck time, and the Planck energy, and such things as Planck acceleration, the first 
four introduced by Max Planck [40] [41] in 1899 and 1906. Max Planck did not say much about exactly what the Planck scale represented, except he thought they represented some important natural units. Einstein [17] in one of his best known general relativity papers published in 1916 suggests that the next step in understanding gravity is a quantum gravity theory, or in his own words:

"Because of the intra-atomic movement of electrons, the atom must radiate not only electromagnetic but also gravitational energy, if only in minute amounts. Since, in reality, this result cannot be the case in nature it appears that the quantum theory must modify not only Maxwell's electrodynamics but also the new theory of gravitation. (Einstein 1916, p. 696)"

Eddington, then in 1918 , is likely the first to speculate that quantum gravity must be linked to the Planck length, or in his own words

three fundamental constants of nature stand out as pre-eminent. The velocity of light, $300 \times 10^{10}$ C.G.S. units; dimensions $\mathrm{LT}^{-1}$. The quantum, $6.55 \times$ $10^{-27}, \mathrm{ML}^{2} \cdot \mathrm{T}^{-1}$ C.G.S. Units. The constant of gravitation, $6.66 \times 10^{-8}$; $\mathrm{M}^{-1} \mathrm{~L}^{2} \cdot \mathrm{T}^{-2}$. From these constants, we can construct a fundamental unit of length whose value is $4 \times 10^{-33} \mathrm{~cm}$. There are other natural units of length, the radii of the positive and negative unit electric charges, but these are of an altogether higher order of magnitude. But this length must be the key to some essential structure. It may not be an unattainable hope that someday a clearer knowledge of the process of gravitation may be reached.

However, this point was not accepted easily and even ridiculed by other prominent physicists like Bridgman [42] in 1931 (Nobel prize winner in physics in 1946, so not exactly a light caliber physicist.) who thought the Planck units were more like mathematical artifacts coming out of dimensional analyses. Today most physicists, in particular, those [43] [44] [45] [46] [47] working with quantum gravity theory assume the Planck scale plays a very important role in a final quantum gravity theory. Still, there are also researchers still with the same view as Bridgman, for example, $[48]^{1}$, that they are mathematical artifacts coming out of the dimensional analysis. To get further in our understanding of the Planck scale is therefore of foremost importance to settle this question in the hope to get forward in quantum gravity theory and unification of gravity and quantum mechanics. We think the recent discovery that the Planck length and Planck time can be measured independent of any knowledge of $G$ or $\hbar$ from simple gravitational observations is a key step forward here, see [37] [38] [39]. But back to the Planck mass. Motz [50] [51] were likely the first to suggest that there could be an important Planck mass particle. However, the Planck mass he naturally knew was enormous compared to the mass of any observed particle and even an atom, so where could the Planck mass be hiding? Motz suggested it could have been created in the big bang and then had radiated its mass into energy that had formed today's known particles such as electrons and protons, ${ }^{1}$ In an otherwise very interesting book. 
and other known elements. The Planck mass particle has also been suggested as the origin of dark matter [46].

Today, the Planck mass is said to be the smallest mass with mathematical properties of a so-called black hole. A black hole is mathematically defined as a mass inside a radius, creating an escape velocity $c$ at this radius. It is often assumed that the mass of a particle is assumed to be inside its Compton wavelength or reduced Compton wavelength, so one possible criterion is that at least the reduced Compton wavelength must be at or inside the Schwarzschild radius [52]. Second, it is often assumed that the Planck length is the smallest possible observable length (even hypothetical). The Schwarzschild radius must be greater or equal to the Planck length.

A point that hardly has been discussed is that general relativity theory cannot match up with the different properties of the Planck scale at the same time. Assume a micro black hole has a Planck mass, if the escape velocity radius is the Planck length, then the escape velocity from general relativity theory is above $c$, that is

$$
v_{e}=\sqrt{\frac{2 G m_{p}}{l_{p}}}=c \sqrt{2} .
$$

This scenario is impossible and is one of several reasons why researchers who have suggested the Planck mass are linked to micro black holes cannot match the full Planck scale, something we will look at in detail here for a series of candidates to micro black holes.

Markov [52] is likely the first already in 1966 to describe that smaller than stellar-mass objects and that even Planck mass size objects can potentially have a gravitationally collapse and the mathematical properties of a black hole, see also [53]. He calculates these collapsed gravitational bodies to have approximately the Planck mass. Based on that, their mass must be inside the Schwarzschild radius. At the same time, the Compton wavelength of the mass must be larger than the Planck length. Hawking ${ }^{2}$ [54] in 1971 that suggested micro black holes only say the micro black hole has a mass approximately equal to the Planck mass. Perhaps he had already seen problems if he put them exactly equal to the Planck mass. Motz and Epstein suggested micro black holes in their 1969 paper [55], they called it mini-black hole that has half the Planck mass. A half Planck mass has an escape velocity of $c$ indeed at the Planck length. Still, then one is not matching the Planck mass and Planck length at the same time, further then both the reduced Compton wavelength and Compton wavelength are outside the Schwarzschild radius, so it do not fit the criteria given by Markov for a gravitationally collapsed object (micro black hole). Faraoni [47] suggests a mass $\sqrt{\pi}$ ${ }^{2} \mathrm{Be}$ aware that, for example, sources like Wikipedia often is very inaccurate when it comes to the history of physics and who came up with what theory first, for example, they incorrectly credit Hawking as the one who first comes up with the idea of micro-black holes in 1971, when Markov is four years ahead of him in publishing such an idea. That Markov's work was published in Russian can naturally have meant it took time for it to be known in the west, but there is no excuse for not updating the page with such proven facts today. 
times the Planck mass that gives a Schwarzschild radius equal to the Compton wavelength, an identical particle we [56] had described already in 2016. In the same working paper, we also mention a $\frac{1}{\sqrt{2}}$ Planck mass size object that has escape velocity $c$ at the reduced Compton wavelength of the particle. However, this mass has then a Schwarzschild radius different from the Planck length, namely $\bar{\lambda}=l_{p} \sqrt{2}$. Obermair [46] suggests a Planck mass black hole and correctly calculates its Schwarzschild radius to be $2 l_{p}$. In other words, none of these matches the different parts of the Planck scale at the same time. With different parts we think about the Planck mass, the Planck time, the Planck length in particular, and perhaps also other Planck units such as the Planck mass acceleration.

Table 1 lists a series of suggested micro-black hole candidates all close to the Planck mass, as we can see none of them match up nicely with the Planck scale. Either one must alter the Planck mass to a mass somewhat different from the Planck mass or alter the Schwarzschild radius to something different from the Planck length. All of these micro black hole candidates except the last mass in the table give a gravitational acceleration at the Schwarzschild radius lower than the Planck acceleration $g_{p}=\frac{G m_{p}}{l_{p}^{2}}$.

More formally, several different criteria can be tried to find the possible best candidate for a micro-black hole. We start with working based on general relativity theory.

Micro black hole that must be exactly equal to the Planck mass and $r_{s} \geq l_{p}$

Assume we say the Planck mass must be a micro-black hole. Its Schwarzschild radius is $r_{s}=\frac{2 G m_{p}}{c^{2}}=2 l_{p}$, so even if it fits the Planck mass, the Schwarzschild radius is different than the Planck length, also its gravitational acceleration at the Schwarzschild radius is

$$
g=\frac{G m_{p}}{r_{s}^{2}}=\frac{G m_{p}}{\left(2 l_{p}\right)^{2}}=\frac{1}{4} \frac{G m_{p}}{l_{p}^{2}}=\frac{1}{4} a_{p} .
$$

So, it also does not match the Planck acceleration, not that anyone says it must. We just point it out at this stage of the article. And again, the escape velocity at the Planck length is higher than $c$. So, the only part of the Planck scale we are able to match perfectly for this micro-black hole is the Planck mass itself.

Micro black hole where the Schwarzschild radius must be equal to the Planck length

Formally we simply set

$$
l_{p}=\frac{2 G m}{c^{2}}
$$

and solve with respect to $m$ to find what mass this micro black hole must have. It 
gives

$$
m=\frac{1}{2} \frac{l_{p} c^{2}}{G}=\frac{1}{2} m_{p}
$$

which is the micro black hole candidate indicated by Motz and Eppstein [55]. Even if it match the Planck length it clearly does not match the Planck mass. It also gives a gravity acceleration different than the Planck acceleration at the Schwarzschild radius, namely

$$
g=\frac{G \frac{1}{2} m_{p}}{l_{p}^{2}}=\frac{1}{2} a_{p}
$$

Further, both the reduced Compton wavelength, $\bar{\lambda}=\frac{\hbar}{m_{p} c}=2 l_{p}$ and the Compton wavelength: $4 \pi l_{p}$, is outside the Schwarzschild radius, so if the mass of a particle spread out the reduced Compton wavelength or the reduced Compton wavelength, then such a mass cannot form a micro black hole.

Micro black hole where the Compton wavelength matches the Schwarzschild radius

Assume the Compton wavelength must be equal to the Schwarzschild radius for the mass to be a micro black hole. To find the mass that gives Schwarzschild radius equal to the Compton wavelength, we can solve the following equation with respect to $x m_{p}$

$$
\frac{2 G x m_{p}}{c^{2}}=\frac{h}{x m_{p} c} .
$$

This result gives

$$
x m_{p}=\frac{\sqrt{c h}}{\sqrt{2 G}}=\sqrt{\pi} m_{p} .
$$

This result corresponds to a candidate for micro black holes as mentioned by Haug [56], and Faraoni [47]. This mass has a Schwarzschild radius equal to $2 \sqrt{\pi} l_{p}$ which is different from the Planck length, and it has an acceleration at the Schwarzschild radius equal to $\frac{a_{p}}{4 \sqrt{\pi}}$ which is different from the Planck acceleration. It also has an escape velocity above $c$ at the reduced Compton wavelength inside the Schwarzschild radius. Still, it is not worse for a candidate for a micro black hole than the other candidates in Table 1, perhaps even among the best candidates. Still, why should there be such a unique mass just somewhat different than the Planck mass, and why should it have such an acceleration different than the Planck acceleration?

Micro black hole where the reduced Compton wavelength matches the Schwarzschild radius

The only mass with a Schwarzschild radius equal to the reduced Compton wavelength under general relativity theory we get by solving the following equa- 
tion with respect to $x m_{p}$

$$
\frac{2 G x m_{p}}{c^{2}}=\frac{\hbar}{x m_{p} c} .
$$

This gives

$$
x m_{p}=\frac{\sqrt{c \hbar}}{\sqrt{2 G}}=\frac{1}{\sqrt{2}} m_{p} .
$$

We suggested this micro-black hole candidate in 2016. We see from the table that this mass has a Schwarzschild radius equal to $r_{s}=l_{p} \sqrt{2}$, so it does not correspond to the Planck length. Also, the acceleration at the Schwarzschild radius is different from the Planck acceleration. Still, this is no worse candidate than the mass that is $\sqrt{\pi} m_{p}$ or the Planck mass itself.

Micro black holes that have Planck acceleration at the Schwarzschild radius

There is only one mass size that has Planck acceleration at the Schwarzschild radius. We can find this mass by solving the equation below with respect to $x m_{p}$

$$
\frac{G \times m_{p}}{\left(\frac{2 G x m_{p}}{c^{2}}\right)^{2}}=\frac{G m_{p}}{l_{p}^{2}}
$$

the solution is

$$
x m_{p}=\frac{c^{4} l_{p}^{2}}{4 G^{2} m_{p}}=\frac{1}{4} m_{p}
$$

so, this result corresponds to the last micro black hole candidate in Table 1. It has a Schwarzschild radius below the Planck length, so it is not consistent with that the smallest possible length can be the Planck length. In addition, both its reduced Compton wavelength and its Compton wavelength are outside the Schwarzschild radius, so parts of its mass would likely be outside the Schwarzschild radius. It can likely not even fit the mathematical criteria for creating a micro-black hole.

None of the micro black hole candidates in Table 1 seems to fit the whole Planck scale, the best candidates are able to match only one (or at best two, candidate 8 ) of the properties of the Planck scale, and one seems to have to choose one of them and then not get a fit to the other properties of the Planck scale. This fact has several implications; it leads to a series of micro-black hole candidates where it seems we do not can decide which one of them is forming a micro-black hole. We could elaborate on this point and even suggest that there could even be a handful of different micro black holes (even before considering such things as rotation).

First when we soon will see micro black holes through the alternative theory, we start to understand that some factor is possibly missing in general relativity theory. Table 2 shows that the Planck mass has all the mathematical properties 
Table 1. Candidates for micro-black holes from general relativity theory, none of them can simultaneously match the Schwarzschild radius and all the Planck scale properties. Only two of these candidates have Schwarzschild radius that correspond either to the Compton wavelength or the reduced Compton wavelength, only a candidate with Schwarzschild radius below the Planck length gives a.

\begin{tabular}{|c|c|c|c|c|c|c|c|c|}
\hline \multicolumn{9}{|c|}{ GENERAL RELATIVITY PREDICTIONS AS WELL AS STANDARD NEWTON } \\
\hline $\begin{array}{c}\text { Mass } \\
\text { candiate }\end{array}$ & $\begin{array}{c}\text { Mass } \\
\text { formula } \\
\text { candiate }\end{array}$ & $\begin{array}{c}\text { Reduced } \\
\text { Compton } \\
\text { wavelength } \\
\bar{\lambda}=\frac{\hbar}{m c}\end{array}$ & $\begin{array}{l}\text { Compton } \\
\text { wavelength } \\
\lambda=\frac{h}{m c}=\bar{\lambda} 2 \pi\end{array}$ & $\begin{array}{c}\text { Schwarzschild } \\
\text { radius } \\
v_{e}=c \\
r_{s}=\frac{2 G m}{c^{2}}\end{array}$ & $\begin{array}{c}\text { Schwarzschild } \\
\text { time } \\
t_{s}=\frac{r_{s}}{c}\end{array}$ & $\begin{array}{c}\text { Escape } \\
\text { velocity at } \\
\text { Compton } \\
v_{e}=\sqrt{\frac{2 G m}{\bar{\lambda}}}\end{array}$ & $\begin{array}{c}\text { Escape } \\
\text { velocity at } \\
\text { redu. Compton } \\
v_{e}=\sqrt{\frac{2 G m}{\lambda}}\end{array}$ & $\begin{array}{c}\text { Acceleration } \\
\text { at } r_{s} \text { where } \\
v_{e}=c \\
g=\frac{G m}{r_{s}^{2}}\end{array}$ \\
\hline $1^{\mathrm{a}}$ & $m_{p}=\sqrt{\frac{\hbar c}{G}}$ & $l_{p}$ & $l_{p} 2 \pi$ & $2 l_{p}$ & $2 t_{p}$ & $\frac{c}{\sqrt{2}}$ & $c \sqrt{2}$ & $\frac{1}{4} a_{p}$ \\
\hline $2^{\mathrm{b}}$ & $\pi m_{p}$ & $\frac{l_{p}}{\pi}$ & $2 l_{p}$ & $2 \pi l_{p}$ & $2 \pi t_{p}$ & $c \sqrt{\pi}$ & $c \sqrt{2} \pi$ & $\frac{a_{p}}{4 \pi}$ \\
\hline $3^{c}$ & $\sqrt{\pi} m_{p}$ & $\frac{l_{p}}{\sqrt{\pi}}$ & $2 \sqrt{\pi} l_{p}$ & $2 \sqrt{\pi} l_{p}$ & $2 \sqrt{\pi} t_{p}$ & c & $c \sqrt{2 \pi}$ & $\frac{a_{p}}{4 \sqrt{\pi}}$ \\
\hline $4^{\mathrm{d}}$ & $2 m_{p}$ & $\frac{l_{p}}{2}$ & $\pi l_{p}$ & $4 l_{p}$ & $4 t_{p}$ & $\frac{2}{\sqrt{\pi}}$ & $\sqrt{8} c$ & $\frac{a_{p}}{8}$ \\
\hline $5^{\mathrm{e}}$ & $\sqrt{2} m_{p}$ & $\frac{l_{p}}{\sqrt{2}}$ & $\sqrt{2} \pi l_{p}$ & $\sqrt{2} l_{p}$ & $\sqrt{2} t_{p}$ & $\frac{\sqrt{2}}{\sqrt{\pi}}$ & $2 c$ & $\frac{a_{p}}{\sqrt{32}}$ \\
\hline $6^{\mathrm{f}}$ & $\frac{1}{\sqrt{2}} m_{p}$ & $\sqrt{2} l_{p}$ & $\sqrt{8} \pi l_{p}$ & $\sqrt{2} l_{p}$ & $\sqrt{2} t_{p}$ & $\frac{c}{\sqrt{2 \pi}}$ & $c$ & $\frac{a_{p}}{\sqrt{8}}$ \\
\hline $7^{g}$ & $\frac{1}{\sqrt{\pi}} m_{p}$ & $\sqrt{\pi} l_{p}$ & $2 \pi^{3 / 2} l_{p}$ & $\frac{2 l_{p}}{\sqrt{\pi}}$ & $\frac{2 t_{p}}{\sqrt{\pi}}$ & $\frac{c \sqrt{2}}{\sqrt{\pi}}$ & $\frac{c}{\pi}$ & $\frac{a_{p} \sqrt{\pi}}{4}$ \\
\hline $8^{\mathrm{h}}$ & $\frac{1}{2} m_{p}$ & $2 l_{p}$ & $4 \pi l_{p}$ & $l_{p}$ & $t_{p}$ & $\frac{c}{2 \sqrt{\pi}}$ & $\frac{c}{\sqrt{2}}$ & $\frac{1}{2} a_{p}$ \\
\hline $9^{\mathrm{i}}$ & $\frac{1}{4} m_{p}$ & $4 l_{p}$ & $8 \pi l_{p}$ & $\frac{1}{2} l_{p}$ & $\frac{1}{2} t_{p}$ & $\frac{c}{\sqrt{16 \pi}}$ & $\frac{c}{\sqrt{8}}$ & $a_{p}$ \\
\hline
\end{tabular}

aSuggested by Obermair [46] 2013 and indicated already by Hawking in 1971 and Markow in 1966, but they only told approximately equal to the Planck mass, likely because they could see it did not fit much of the Planck scale, so perhaps there was a better candidate? ${ }^{b}$ Likely first suggested in this paper. ${ }^{\mathrm{c}}$ Suggested by Haug [56] in 2016 and Faraoni [47] in 2017. 'Suggested by Markov [52] in 1967. ${ }^{\mathrm{e}}$ Likely first suggested in this paper. ${ }^{\mathrm{f}}$ Suggested by [56] in 2016. ${ }^{\mathrm{g}}$ First time suggested here we think. ${ }^{\mathrm{h}}$ Suggested by Motz and Epstein [55] in 1979. ${ }^{\mathrm{i}}$ Likely first suggested in this paper.

Table 2. The Planck mass perfectly fits all the mathematical properties of a micro-black hole in our new model, which is all aspects of the Planck scale.

\begin{tabular}{|c|c|c|c|c|c|c|c|c|}
\hline \multicolumn{9}{|c|}{ COLLISION SPACE-TIME QUANTUM GRAVITY, THE METRIC PRESENTED IN THIS PAPER } \\
\hline Mass & $\begin{array}{c}\text { Mass } \\
\text { formula }\end{array}$ & $\begin{array}{c}\text { Reduced } \\
\text { Compton } \\
\text { wavelength } \\
\bar{\lambda}=\frac{\hbar}{m c}\end{array}$ & $\begin{array}{l}\text { Compton } \\
\text { wavelength } \\
\lambda=\frac{h}{m c}=\bar{\lambda} 2 \pi\end{array}$ & $\begin{array}{l}\text { Escape } \\
\text { radius } \\
v_{e}=c \\
r_{c}=\frac{G m}{c^{2}}\end{array}$ & $\begin{array}{l}\text { Escape } \\
\text { radius } \\
\text { time } \\
t_{c}=\frac{r_{c}}{c}\end{array}$ & $\begin{array}{c}\begin{array}{c}\text { Escape } \\
\text { velocity at } \\
\text { Compton }\end{array} \\
v_{e}=\sqrt{\frac{2 G m}{\bar{\lambda}}-\frac{G^{2} m^{2}}{c^{2} \bar{\lambda}^{2}}}\end{array}$ & $\begin{array}{c}\begin{array}{c}\text { Escape } \\
\text { velocity at } \\
\text { redu. Compton }\end{array} \\
v_{e}=\sqrt{\frac{2 G m}{\lambda}-\frac{G^{2} m^{2}}{c^{2} \lambda^{2}}}\end{array}$ & $\begin{array}{c}\text { Acceleration } \\
\text { at } r_{c} \text { where } \\
v_{e}=c \\
g=\frac{G M}{r_{c}^{2}}\end{array}$ \\
\hline Planck mass & $m_{p}=\sqrt{\frac{\hbar c}{G}}$ & $l_{p}$ & $l_{p} 2 \pi$ & $l_{p}$ & $t_{p}$ & $\approx 0.54 c$ & $c$ & $a_{p}$ \\
\hline
\end{tabular}

of a micro black holes and that all the elements of the Planck scale elements fit in when we are working out from our new escape velocity and its corresponding 
space-time metric. Then for the Planck mass, suddenly, the radius where the escape velocity is $c$ is the Planck length, which is then also identical to the reduced Compton wavelength of the Planck mass. Further, we get a Planck mass acceleration at the radius where the escape velocity is $c$. Also, what we can call the escape radius time is the Planck time.

Also, note that in our model, the acceleration at the radius where $v_{e}=c$ during the Planck time will bring the velocity to $c$, that is simply $a_{p} t_{p}=c$, the importance of this point we have discussed somewhat in [4]. Also, the time it takes light to cross the Planck mass, reduced Compton wavelength, and the time the light to cross the radius where the escape velocity is $c$ is the Planck time. In other words, we have a perfect match to the Planck time, the Planck length and the Planck mass, the Planck acceleration, and we will also see the Planck energy (temperature).

In our collision space-time model, the Planck mass particle that corresponds to the mathematical framework of a micro black-hole is simply the collision point between the building blocks of two photons. The collision between two such building blocks lasts the Planck time for them to leave each other at the speed of light. They are mass (the Planck mass) when colliding but are massless and moving at speed $c$ when not colliding, see the just mentioned paper for an in-depth discussion on this topic. In our model, all masses are created by Planck mass particles. This mass is the only pure mass. The Planck mass particle only lasts for the Planck time and comes into existence at the reduced Compton frequency of the particle. For example, an electron mass is given by

$$
m_{e}=\frac{c}{\bar{\lambda}_{e}} m_{p} t_{p}=\frac{\hbar}{\bar{\lambda}_{e}} \frac{1}{c}=9.11 \times 10^{-31} \mathrm{~kg}
$$

but for an in-depth discussion on this point, see the papers referred to about collision space-time. Our new theory perfectly match up with the Planck scale and how it is linked to the mathematical properties of so-called micro-black holes. It also tells us where this Planck mass is. It is inside every mass. It pops in and out of existence at the reduced Compton frequency of matter. Assume we only have one collision between indivisible particles. This result is a Planck mass lasting one Planck time, so its mass is $m_{p} t_{p} \approx 1.17 \times 10^{-51} \mathrm{~kg}$ (seconds). This result is very close to the photon mass, as suggested by several authors in the literature. See [12] for a review. However, in our theory, the Planck mass is very small. To observe it as a Planck mass directly, one has to observe it inside the lifetime of the particle, namely the Planck time. Then the mass is the Planck mass. However, when observed over a second observational time-window, it is only $m_{p} t_{p}$ see [2] [3] [4] for an in-depth discussion on this topic. In our theory, we know where the particle is hiding that fits the mathematical properties of a micro black hole, it is inside all matter, it also gives the mass of a photon (the building block of a photon), it answers the mass-gap (it is the mass-gap), it gives a better understanding of photons and light. But there is more to it. The micro-black hole is not a black hole at all. It is a fully solid of two indivisible particles colliding. Let 
us also try to solve the escape velocity formula for $r$ when $v_{e}=0$, in other words, a special case. In general relativity theory, we would have

$$
v_{e}=0=\sqrt{\frac{2 G M}{r}} .
$$

This problem has no solution. Some researchers will possibly say that is okay as it makes no sense to have an escape velocity of 0 as it would not be a velocity, but something standing still, but please keep an open mind. In our model, we have

$$
v_{e}=0=\sqrt{\frac{2 G M}{r}-\frac{G^{2} M^{2}}{c^{2} r^{2}}}
$$

solved with respect $r$ this gives $r=\frac{G M}{2 c^{2}}$. This result is half of the radius where the escape velocity is $c$. We do not think this fact adds much interpretation to black holes in general, except for the Planck mass particle (micro black hole). This result means that halfway inside the radius where $v_{e}=c$, our model says that what is there must be standing still. Our theory also describes the interior of the micro-black hole. That is halfway inside the Planck length radius. There is an object standing, it has velocity $v=0$. This result sounds like it is against the relativity principle because it must be moving against some reference frame? A key point, as discussed in detail by [3] is that if the Planck mass particle simply consists of the two photons (building blocks) that are colliding for one Planck time, and have a Planck length diameter each. Then this object can only be directly observed from itself. This result is exactly our model that a photon-photon collision consists of two indivisible particles, each with diameter $l_{p}$ stands still when colliding for the Planck time, to escape each other at the speed of light. Halfway inside the radius where the escape velocity is $c$ is the center of the indivisible particle. The two indivisible particles stand still in the collision that only can be observed directly from itself as it dissolves before any signal can get into it or out from it. Each indivisible particle that at this moment are standing still extends from the center of this object (collision) to the circumference of this object. The circumference has a radius equal to the Planck length. And it is important to be aware that we do not assume the diameter of the indivisible particle is the Planck length. This result we get from calibrating our model to observable gravity phenomena as described in great detail in [2] [4]. Even if the Planck mass particle and, therefore, the micro black hole cannot be observed directly, we can observe them indirectly through what they are causing. That is gravity itself, as described in the papers just mentioned. This result is also why we recently have been able to understand how to measure the Planck scale (the Planck length, the Planck time, and the Planck mass) independent of any knowledge of $G$ [38]. There is no longer a need to search for how to detect the Planck scale. It is detected in every observation of gravity. Even if already published in decent peer-reviewed journals, such claims should naturally be scrutinized by many researchers before a final conclusion is made. Still, that our theory so well matches 
the Planck scale at the same time it matches criteria for micro black holes, something we have demonstrated general relativity theory does not do, should hopefully at least make more researchers curious about this theory.

Also, be aware that the Planck mass acceleration can maximum last the Planck time to keep the whatever is accelerating not to reach superluminal speeds because

$$
a_{p} t_{p}=\frac{G m_{p}}{l_{p}^{2}} \frac{l_{p}}{c}=c
$$

Only photons can be accelerated to speed $c$ as no mass can move at speed $c$. So, this result fits our theory perfectly where the Planck mass (a micro black-hole) simply is the collision of two indivisible particles (photons). And this collision lasts only the Planck time, again by calibration to gravity phenomena, not by assumption. In standard theory, the Planck mass acceleration cannot exist if we also want to hold on to that Planck time and the Planck length are minimum units. In that theory, photons cannot stand still even when colliding with each other. So how can then anything in the standard theory match up with the Planck acceleration? This result is only possible if something goes from rest-mass to light within the Planck time. In our theory colliding indivisible particles are mass, and non-colliding indivisible particles move at the speed of light $c$. This result needs to match the Planck acceleration, and our theory does so. The Planck acceleration does not seem to be fully consistent with standard theory, as no mass can be accelerated to $c$. Naturally, the standard theory could claim the Planck mass radiated into energy within the Planck time after the big bang or something like that. However, such a totally non-testable hypothesis is not needed. We think the Planck mass acceleration indicates that only indivisible particles (photons) can go into the Planck mass. They create the Planck mass, as the Planck mass in our model is a collision of two such indivisible particles, and only light can come out from the Planck mass (micro black hole), no mass.

Table 3 summarizes some of our findings about the Planck mass given in this paper and related papers on collision space-time. The Planck mass seems to be the key to understanding the link between photons and mass, the mass gap, gravity at the quantum level. It is the very essence of our universe.

\section{Radiation from "Black Holes"}

While the Hawking radiation is given by

$$
T=\frac{\hbar c^{3}}{8 \pi G k_{b} M}=\frac{T_{p}}{8 \pi}=\frac{M c^{2}}{8 \pi k_{b}} .
$$

Our framework seems it will give

$$
T=\frac{\hbar c^{3}}{2 \pi G k_{b} M}=\frac{T_{p}}{2 \pi}=\frac{M c^{2}}{2 \pi k_{b}} .
$$

In the case of a micro black hole, this result means the radiation is 
Table 3. Summary of findings about the Planck mass in this paper and related papers rooted in collision space-time.

\begin{tabular}{ll}
\hline Planck mass fits properties "black-hole" & Yes \\
Planck length fits properties "black-hole" & Yes \\
Planck time fits properties "black-hole" & Yes \\
Planck acceleration fits properties "black-hole" & Yes \\
Planck energy fits properties "black-hole" & Yes \\
Can we detect the Planck mass particle, it is gravity" & Yes \\
Lifetime of Planck particle & $t_{p}$ \\
Lifetime of micro black hole & $t_{p}$ \\
Velocity inside of micro black hole & $v_{e}=0$ \\
$a_{p} t_{p}=c$ fits logic & yes \\
Photon mass & $m_{p} t_{p}$ and $m_{p}$ \\
Explains the mass gap & yes \\
Planck mass is building blocks of all masses & yes \\
What is the Planck mass & a photon-photon collision \\
What is the Planck mass & a micro-black solid lasting for the Planck time \\
Planck scale incorporated in quantum mechanics & which is the same as a photon-photon collision. \\
Planck scale incorporated in quantum gravity & yes \\
\hline
\end{tabular}

${ }^{\mathrm{a}}$ See $\left[\right.$ Haug(2020)]; ${ }^{\mathrm{b}}$ see reference above; ${ }^{\mathrm{c}}$ see reference above; ${ }^{\mathrm{d}}$ see reference above.

$$
T=\frac{m_{p} c^{2}}{2 \pi k_{b}}=\frac{T_{p}}{2 \pi}
$$

The $2 \pi$ here has to do with assuming that the mini black-hole's geometry (solid) is spherical. Actually, it is, in our view, not spherical. However, simply two indivisible spheres colliding for then to moving away from each other again, and the radiation is therefore only in two directions, the two indivisible particles leaving each other, this result gives mini-black hole radiation of $T=\frac{E_{p}}{k_{b}}=\frac{m_{p} c^{2}}{k_{b}}$ which is the Planck mass-energy. The micro-black hole is the Planck mass. It dissolves into the Planck mass-energy.

\section{Hubble Scale}

Our theory also gives a series of new predictions around the Hubble scale. For example it gives a critical mass of the observable universe that is twice of that as is predicted by the Friedman equation. That is the critical mass in our theory is given by

$$
M_{c}=\frac{c^{3}}{G H_{0}}
$$


versus the critical mass from general relativity theory which is

$$
M_{c}=\frac{c^{3}}{2 G H_{0}} .
$$

Interestingly the reduced Compton frequency per Planck time from our new critical universe mass multiplied by the Planck time is equal to the Hubble time. That is we have

$$
T_{H}=\frac{l_{p}}{\bar{\lambda}_{c}} t_{p} \approx 13.7 \text { billion years. }
$$

While in general relativity theory we will have

$$
T_{H}=\frac{2 l_{p}}{\bar{\lambda}_{c}} t_{p} \approx 13.7 \text { billion years }
$$

further we have that the Hubble radius in our new theory is equal to

$$
R_{H}=\frac{l_{p}}{\bar{\lambda}_{c}} l_{p}
$$

while in general relativity theory we get

$$
R_{H}=\frac{2 l_{p}}{\bar{\lambda}_{c}} l_{p} .
$$

Just as general relativity theory do not seem to fit well with the Planck scale in relation to micro black holes, it also seems like something very similar is going on in relation to the Hubble scale of the universe when tried linked to the Planck scale, general relativity theory. Be aware that both the Friedman equation for the critical universe and our new model for the critical universe can be derived from escape velocity alone [57]. For more on the Hubble scale in relation to our new theory see this paper.

\section{Conclusion}

We have suggested a parallel to the Schwarzschild metric for our collision space-time theory that seems to be linked to a three-dimensional space-time, three dimensions in space and three in time that are closely linked. This metric gives a much better fit and understanding of the Planck scale. It has a theory behind it that is able to unify gravity with quantum mechanics. There are certainly much more to investigate in collision space-time, but even at this stage, we think it should be of great interest to the physics community to investigate this theory further.

\section{Conflicts of Interest}

The author declares no conflicts of interest regarding the publication of this paper.

\section{References}

[1] Minkowski, H. (1908) Space and Time. The 80 th Assembly of German Natural 
Scientists and Physicians, Cologne, 21 September 1908, 133-216.

[2] Haug, E.G. (2012) Rethinking the Foundation of Physics and Its Relation to Quantum Gravity and Quantum Probabilities: Unification of Gravity and Quantum Mechanics. https://vixra.org/abs/2012.0089

[3] Haug, E.G. (2021) Quantum Gravity Hidden in Newton Gravity and How to Unify It with Quantum Mechanics. In: The Origin of Gravity from the First Principles, NOVA Publishing, New York.

[4] Haug, E.G. (2020) Collision Space-Time: Unified Quantum Gravity. Physics Essays, 33, 46. https://doi.org/10.4006/0836-1398-33.1.46

[5] Cole, E.A.B. (1980) Particle Decay in Six-Dimensional Relativity. Journal of Physics A: Mathematical and General, 13, 109. https://doi.org/10.1088/0305-4470/13/1/012

[6] Ziino, G. (1981) Three-Dimensional Time and Thomas Precession. Lettere al Nuovo Cimento, 31, 629-632. https://doi.org/10.1007/BF02777968

[7] Cole, E.A.B. (1983) A Proposed Observational Test of Six-Dimensional Relativity. Journal of Physics A: Mathematical and General, 85, 282. https://doi.org/10.1016/0375-9601(83)90019-1

[8] Boyling, J.B. and Cole, E.A.B. (1993) Six-Dimensional Dirac Equation. International Journal of Theoretical Physics, 32, 801-812. https://doi.org/10.1007/BF00671667

[9] Lanciani, P.A. (1999) Model of the Electron in a 6-Dimensional Spacetime. Foundations of Physics, 29, 251-265. https://doi.org/10.1023/A:1018825722778

[10] Pilotti, J. (2019) How Minkowski Could Have Discovered Six Dimensional Spacetime. Second Hermann Minkowski Meeting, Albena, 13-16 May 2019, 463. http://www.minkowskiinstitute.org/meetings/2019/Abstracts/Pilotti.pdf

[11] Cole, E.A.B. (1977) Superluminal Transformations Using Either Complex Space-Time or Real Space Time Symmetry. Il Nuovo Cimento A, 40, 171-180. https://doi.org/10.1007/BF02776784

[12] Spavieri, G., Quintero, J., Gilles, G.T. and Rodriguez, M. (2011) A Survey of Existing and Proposed Classical and Quantum Approaches to the Photon Mass. The European Physical Journal D, 61, 531-550. https://doi.org/10.1140/epjd/e2011-10508-7

[13] Sommerfeld, A. (1948) Elekrodynamik, Vorlesnungen über Theoretische Physik: Band III. Dieterich.

[14] Rindler, W. (2001) Relativity, Special, General and Cosmology. Second Edition, Oxford University Press, Oxford.

[15] Schwarzschild, K. (1916) Über das gravitationsfeld eines massenpunktes nach der einsteinschen theorie. Sitzungsberichte der Deutschen Akademie der Wissenschaften zu Berlin, Klasse fur Mathematik, Physik, und Technik, 189.

[16] Schwarzschild, K. (1916) Über das gravitationsfeld einer kugel aus inkompressibler flussigkeit nach der einsteinschen theorie. Sitzungsberichte der Deutschen Akademie der Wissenschaften zu Berlin, Klasse fur Mathematik, Physik, und Technik, 424.

[17] Einstein, A. (1916) Näherungsweise integration der feldgleichungen der gravitation. Sitzungsberichte der Königlich Preussischen Akademie der Wissenschaften Berlin.

[18] Augousti, A.T. and Radosz, A. (2006) An Observation on the Congruence of the Escape Velocity in Classical Mechanics and General Relativity in a Schwarzschild Metric. European Journal of Physics, 376, 331-335. https://doi.org/10.1088/0143-0807/27/2/015

[19] Guifry, M. (2019) Modern General Relativity. Cambridge University Press, Cambridge. 
[20] Haug, E.G. (2021) Demonstration That Newtonian Gravity Moves at the Speed of Light and Not Instantaneously (Infinite Speed) as Thought! Journal of Physics Communication, 5, Article ID: 025005.

[21] Lan, S., Kuan, P., Estey, B., English, D., Brown, J.M., Hohensee, M.A. and Müller (2013) A Clock Directly Linking Time to a Particle's Mass. Science, 339, 554-557. https://doi.org/10.1126/science.1230767

[22] Dolce, D. and Perali, A. (2015) On the Compton Clock and the Undulatory Nature of Particle Mass in Graphene Systems. The European Physical Journal plus, 130, Article No. 41. https://doi.org/10.1140/epjp/i2015-15041-5

[23] Rindler, W. (1960) Special Relativity. Oliver and Boyd, Edinburgh.

[24] Rindler, W. (2006) Relativity, Special, General and Cosmology. Second Edition, Oxford University Press, Oxford.

[25] Giancoli, D.C. (2009) Physics for Scientists \& Engineers. Pearson Prentice Hall, Hoboken.

[26] Tipler, P.A. and Llewellyn, R.A. (1999) Modern Physics. Third Edition, W.H. Freeman and Company, New York.

[27] Grøn, Ø. (2020) Introduction to Einstein's Theory of Relativity, from Newton's Attractive Gravity to the Repulsive Gravity of Vacuum Energy. Second Edition, Springer Verlag, Berlin.

[28] Born, M. (1920) Die Relativitätstheorie Einsteins und ihre physikalischen Grundlagen (Einstein's Theory of Relativity, Translated to English 1965, Dover). Springer, Berlin.

[29] Adler, C.G. (1987) Dose Mass Really Depends on Velocity Dad? American Journal of Physics, 55, 739-743.

[30] Okun, L.B. (1989) The Concept of Mass. Physics Today, 42, 31. https://doi.org/10.1063/1.881171

[31] Hecht, E. (2009) Einstein Never Approved the Relativistic Mass Formula. The Physics Teacher, 47, 336-341. https://doi.org/10.1119/1.3204111

[32] Taylor, E.F. and Wheeler, J.A. (1992) Spacetime Physics, Introduction to Special Relativity. W. H. Freeman and Company, New York.

[33] Rindler, W. (1990) Putting to Rest Mass Misconceptions. Physics Today, 43, 13-15. https://doi.org/10.1063/1.2810555

[34] Jammer, M. (2000) Concepts of Mass in Contemporary Physics and Philosophy. Princeton University Press, Princeton.

[35] Haug, E.G. (2016) The Gravitational Constant and the Planck Units. A Simplification of the Quantum Realm. Physics Essays, 29, 558.

https://doi.org/10.4006/0836-1398-29.4.558

[36] Haug, E.G. (2016) Planck Quantization of Newton and Einstein Gravitation. International Journal of Astronomy and Astrophysics, 6, 206-217.

https://doi.org/10.4236/ijaa.2016.62017

[37] Haug, E.G. (2017) Can the Planck Length Be Found Independent of Big G? Applied Physics Research, 9, 58.

[38] Haug, E.G. (2020) Finding the Planck Length Multiplied by the Speed of Light without Any Knowledge of $g$, $c$, or $h$, Using a Newton Force Spring. Journal Physics Communication, 4, Article ID: 075001.

[39] Compton, A.H. (1923) A Quantum Theory of the Scattering of X-Rays by Light Elements. Physical Review, 21, 483-502. https://doi.org/10.1103/PhysRev.21.483 
[40] Planck, M. (1899) Natuerliche Masseinheiten. Der Königlich Preussischen Akademie Der Wissenschaften.

[41] Planck, M. (1906) Vorlesungen über die Theorie der Wärmestrahlung. J.A. Barth, Leipzig, 163. See Also the English Translation "The Theory of Radiation" (1959) Dover.

[42] Bridgman, P.W. (1931) Dimensional Analysis. Yale University Press, New Haven.

[43] Padmanabhan, T. (1985) Planck Length as the Lower Bound to All Physical Length Scales. General Relativity and Gravitation, 17, 215-221. https://doi.org/10.1007/BF00760244

[44] Hossenfelder, S. (2012) Can We Measure Structures to a Precision Better than the Planck Length? Classical and Quantum Gravity, 29, Article ID: 115011.

[45] Hossenfelder, S. (2013) Minimal Length Scale Scenarios for Quantum Gravity. Living Reviews in Relativity, 16, Article No. 2.

[46] Obermair, G.M. (2013) Primordial Planck Mass Black Holes (PPMBHs) as Candidates for Dark Matter? Journal of Physics, Conference Series, 442, Article ID: 012066 .

[47] Faraoni, V. (2017) Three New Roads to the Planck Scale. American Journal of Physics, 85, 865-869. https://doi.org/10.1119/1.4994804

[48] Unzicker, A. (2020) The Mathematical Reality: Why Space and Time Are an Illusion.

[49] Haug, E.G. (2021) Using a Grandfather Pendulum Clock to Measure the World's Shortest Time Interval, the Planck Time (with Zero Knowledge of G). Journal of Applied Mathematics and Physics, 9, 1076-1088. https://doi.org/10.4236/jamp.2021.95074

[50] Motz, L. (1962) Gauge Invariance and the Structure of Charged Particles. Il Nuovo Cimento (1955-1965), 260, 672-697. https://doi.org/10.1007/BF02781795

[51] Motz, L. (1966) A Gravitational Theory of the Mu Meson and Leptons in General. Rutherford Observatory, Columbia University, New York.

[52] Markov, M.A. (1967) Elementary Particles of Maximally Large Masses (Quarks and Maximons). Soviet Physics JPT, 24, 584.

http://inis.jinr.ru/sl/NTBLIB/JINR-E2-2973.pdf

[53] Markov, M.A. (1987) The "Maximon" and "Minimon" in Light of a Possible Formulation of the Concept of an "Elementary Particle". Soviet Physics JPT, 45, 143. http://jetpletters.ru/ps/1237/article_18690.shtml

[54] Hawking, S. (1971) Gravitationally Collapsed Objects of Very Low Mass. Monthly Notices of the Royal Astronomical Society, 152, 75-78.

https://doi.org/10.1093/mnras/152.1.75

[55] Motz, L. and Epstein, J. (1979) The Gravitational Charge $1 / 2 \sqrt{\mathrm{hc}}$ as a Unifying Principle in Physics. Il Nuovo Cimento, 51, 88-113. https://doi.org/10.1007/BF02822327

[56] Haug, E.G. (2016) The Planck Mass Particle Finally Discovered! The True God Particle! Good Bye to the Point Particle Hypothesis! http://vixra.org/abs/1607.0496

[57] Haug, E.G. (2021) A New Full Relativistic Escape Velocity and a New Hubble Related Equation for the Universe. https://hal.archives-ouvertes.fr 